\title{
SOFT X-RAY EMISSION FROM CLASSICAL NOVAE IN OUTBURST
}

\author{
Sumner Starrfield \\ IGPP and Theoretical Division, Los Alamos National Laboratory \\ and Department of Physics and Astronomy, Arizona State University \\ James W. Truran \\ Department of Astronomy, University of Illinois \\ Warren M. Sparks \\ Applied Theoretical Division, Los Alamos National Laboratory \\ Joachim Krautter \\ Landessternwarte, Königstuhl \\ James MacDonald \\ Department of Physics and Astronomy, University of Delaware
}

\begin{abstract}
Theoretical modeling of novae in outburst predicts that they should be active emitters of radiation at soft X-ray wavelengths twice during their outburst. The first time occurs very early in the outburst when only a very sensitive all sky survey will be able to detect them. This period lasts only a few hours for the very fastest novae. They again become bright in $\mathrm{X}$-rays late in the outburst when the remnant object becomes very hot and is still luminous. Both simulations and observations show that novae can remain very hot for months to years. It is important to observe them at these late times because a measurement both of the flux and temperature can provide information about the mass of the white dwarf, the turn-off time scale, and the energy budget of the outburst.
\end{abstract}

\section{INTRODUCTION}

The observational studies of novae have shown that, during their outbursts, they emit energy over a very broad range in wavelengths. In this paper, we assume the accepted model for the classical nova outburst and show how this model predicts that novae will be sources of both soft X-ray and EUV emission twice during their outbursts. Briefly, the model for the outburst involves a white dwarf star that is a close binary companion to a larger, cooler, star which fills its Roche Lobe. For reasons that are as yet unclear, the cooler star loses mass through the inner Lagrangian point into the lobe surrounding the white dwarf. This material falls into an accretion disk and eventually onto the white dwarf. Over a long period of time the accreting material (usually found to be hydrogen-rich) grows in thickness until the conditions at the boundary between the accreted material and the core material are such that a thermonuclear runaway is 
initiated in the envelope. Reviews of this mechanism can be found in Starrfield (1989) and Shara (1989). Here we concentrate on the effects of the runaway on the emission at the surface of the white dwarf.

Since the outburst is an explosion occurring on a white dwarf star, it is clear that if the white dwarf's luminosity is close to the Eddington luminosity when its radius is small, then soft X-ray emission must occur. On the other hand, if the white dwarf has a larger radius, then peak $\mathrm{T}_{e f f}$ will be lower even if it is radiating at $\mathrm{L}_{E d d}$.

\section{THE RISE AND FALL OF SOFT X-RAY EMISSION IN NOVAE}

All of the hydrodynamic calculations have shown that the rise time of the thermonuclear runaway is very fast and occurs on an exponential time scale (Truran 1982, Starrfield 1989) since the rate of nuclear energy generation depends upon a high power of the temperature. In addition, because the nuclear reactions are occurring in partially electron- degenerate material, there is little expansion in the envelope until the temperature in the shell source exceeds the Fermi temperature.

However, as the temperature in the shell source grows past $\sim 30 \times 10^{6} \mathrm{~K}$, a convective region forms just above the region of peak burning and carries most of the nuclear energy toward the surface. As the temperatures continue to rise, the size of the convective region also grows and shortly after the temperature in the shell source exceeds $\sim 10^{8} \mathrm{~K}$, convection reaches the surface of the white dwarf star. This has two important effects. First, it carries most of the nuclear energy to the surface on a time scale that is much faster than the envelope can adjust to the heat being deposited in the outer layers. Second, convective transport also carries the $\beta^{+}$-unstable nuclei to the surface where they decay and cause the local rate of nuclear energy generation to exceed $10^{12} \mathrm{erg} \mathrm{gm}^{-1}$ $\mathrm{sec}^{-1}$. This intense energy release in the outer layers causes the luminosity to jump to $\mathrm{L}_{E d d}$, or even beyond, on the convective turn-over time scale which, for a white dwarf, is about an hour. If we assume that no radius expansion has occurred during the growth of the thermonuclear runaway to peak temperature so that the star reaches $\mathrm{L}_{E d d}$ when it still has the equilibrium radius of a white dwarf, then we arrive at the curve shown in Figure 1. Since the radius is a strongly decreasing function of white dwarf mass, peak $\mathrm{T}_{e f f}$ is a strongly increasing function of white dwarf mass. In fact, of course, there will be some expansion of the white dwarf during the evolution to the outburst and the actual peak temperatures reached in the numerical simulations are somewhat less. They are also displayed in Figure 1 as points at four different white dwarf masses and come from various simulations that we have computed. Although each point falls below the curve, it is clear that the most massive white dwarfs can reach peak $\mathrm{T}_{\text {eff }}$ exceeding $\sim 1.2 \times 10^{6} \mathrm{~K}$. Therefore, just as the white dwarf reaches peak $\mathrm{M}_{b o l}$ it will also be bright in soft $\mathrm{X}$-rays.

Unfortunately, this stage is very short-lived as can be seen in Figure 2 which shows the result for one simulation at a white dwarf mass of $1.35 \mathrm{M}_{\odot}$. The intense energy deposition into the envelope causes a very rapid expansion of the outer layers and a concomitant decrease in $\mathrm{T}_{\text {eff }}$. It is possible that such an outburst could be detected by a very sensitive all sky survey and would appear as a very soft $X$-ray transient. 


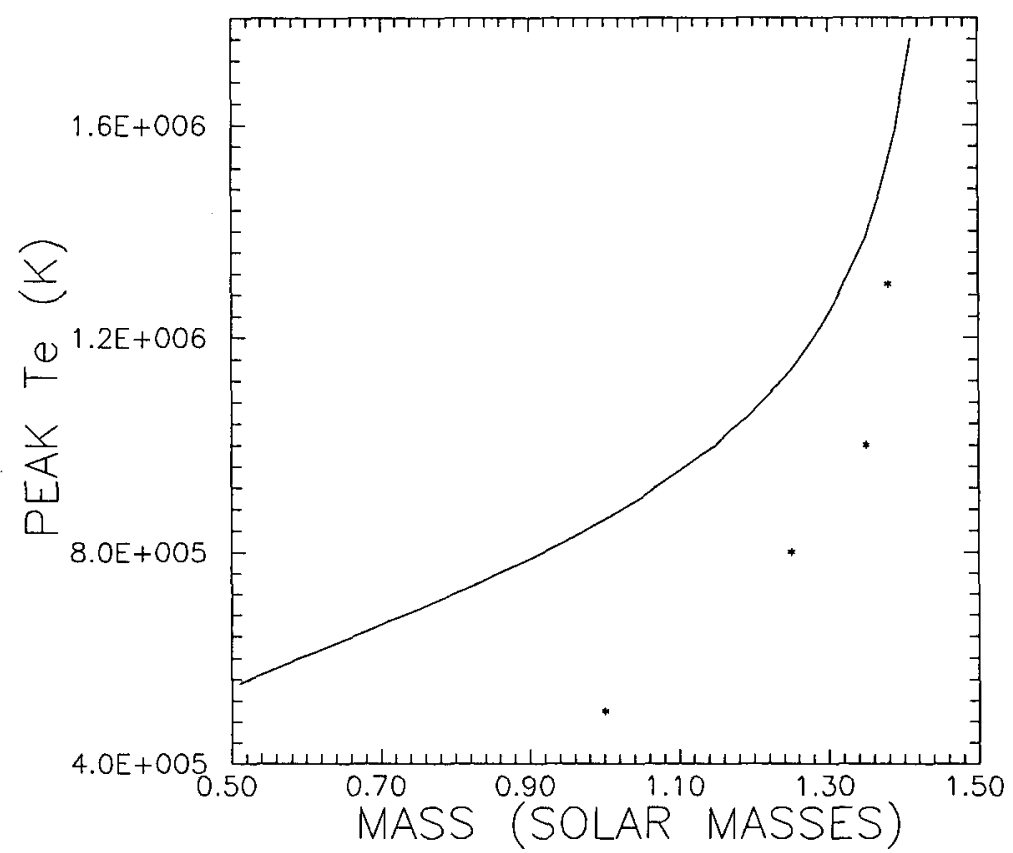

Figure 1 . The peak $T_{e}$ for a white dwarf radiating at the Eddington luminosity versus mass. The points are from simulations at $1.0 \mathrm{M}_{\odot}, 1.25 \mathrm{M}_{\odot}$, $1.35 \mathrm{M}_{\odot}$, and $1.38 \mathrm{M}_{\odot}$.

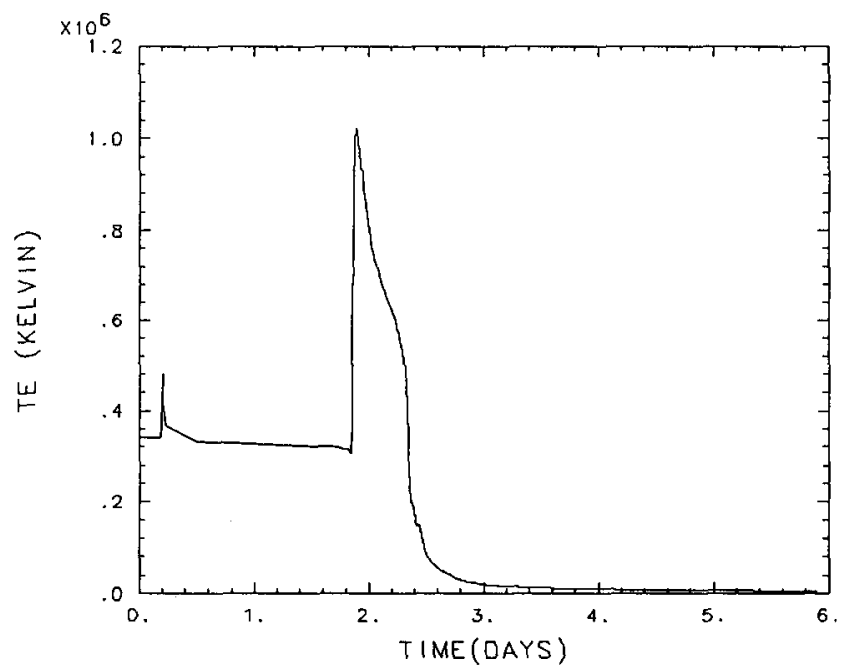

Figure 2 The variation of $T_{e}$ as a function of time near peak luminosity for a $1.35 \mathrm{M}_{\odot}$ simulation. The rapid decrease after maximum is caused by the expansion of the outer layers. 
Once the outer layers begin to expand, the effective temperature declines steadily until the expanding envelope reaches temperatures of $\sim 10^{4} \mathrm{~K}$. At this time the outer layers of the expanding envelope become optically thin and the pseudo-photosphere moves inward in mass to deeper and hotter layers.. In addition, observations show and theory predicts that the shell source continues to produce energy at very high rates keeping the evolving nova radiating at near $\mathrm{L}_{E d d}$. This constant luminosity phase was one of the first predictions of the thermonuclear runaway theory to be confirmed by observations (Starrfield et al. 1972; Gallagher and Code 1974). The simulations show that it will last until almost the entire accreted envelope has been ejected or burned.

The intense heating of the outer layers plus the fact that the secondary is orbiting within the outer layers of the rekindled white dwarf (the common envelope phase) drives a period of rapid mass loss. The resulting evolution of the white dwarf at this time is to smaller radii at constant luminosity. Hence, the effective temperature begins to increase to values exceeding $10^{5} \mathrm{~K}$. The white dwarf will continue to evolve, with decreasing radius and increasing $\mathrm{T}_{\text {eff }}$, until it has again reached its equilibrium radius and the outburst ends. The $\mathrm{T}_{\text {eff }}$ of the white dwarf will move vertically in Figure 1 until it reaches the smooth curve. Thus, there will be a second period of soft $\mathrm{X}$-ray emission during the outburst (depending upon the mass of the white dwarf).

The time scale for this phase of the outburst will also depend upon the mass of the white dwarf. We remind the reader that the amount of envelope mass necessary to produce a runaway is a decreasing function of white dwarf mass, while the rate of mass loss during the constant luminosity phase is an increasing function of white dwarf mass. Therefore, the time to return the white dwarf to quiescence must be a very strongly decreasing function of white dwarf mass. Current estimates for rates of mass loss, which depend only on radiation pressure driven ejection, imply that the time scale to rid the white dwarf of its entire accreted envelope can be less than a year for the most massive white dwarfs.

\section{SUMMARY AND DISCUSSION}

We have demonstrated that classical novae should produce soft X-ray emission twice during the outburst. The first phase occurs very early in the observable part of the outburst when the energy from the shell source first reaches the surface. At this time the luminosity is at or exceeds $\mathrm{L}_{E d d}$ and the radius is still close to that of an equilibrium white dwarf. One problem with this prediction is that there is evidence that a fraction of novae show a gradual rise in visual magnitude for some time before the last sharp rise to maximum (Robinson 1975). If this early rise is an indication of interior heat reaching the surface before the peak of the runaway, then it would seem likely that the radius of the white dwarf has grown and that EUV rather than soft X-ray emission will occur at early phases. It would be interesting to find the cause of the slow rise.

The second period of soft X-ray and EUV emission occurs long after maximum and is a direct result of the constant luminosity of the white dwarf in combination with the gradually shrinking envelope (as mass is lost). The important point about this phase 
is that it will last a long time and there is now observational evidence to support this prediction. Nova GQ Mus 1983 was detected in X-rays by EXOSAT and now shows the $[\mathrm{Fe} \mathrm{X}]$ coronal line stronger than $\mathrm{H} \alpha$ (Krautter and Williams 1989). IUE spectra of this same nova show a very blue continuum (Starrfield et al. 1989) that is also indicative of a high temperature white dwarf. The hydrodynamic simulations of the outburst show that almost the entire accreted envelope (plus material mixed into the envelope from the core) must be ejected in order for the system to return to quiescence and the white dwarf to an equilibrium radius. Therefore, in the final stages of the outburst the effective temperature of the white dwarf should climb to the curve plotted in Figure 1. Finally, we also note that the time scale for turnoff and the peak temperature reached by the nova in the last stages of the outburst are a strong function of the mass of the white dwarf.

We would like to express our thanks for many useful discussion to Drs. G. S. Kutter, H. Ögelman, R. Polidan, G. Shaviv, S. Shore, E. M. Sion, L. Stryker, G. Sonneborn, R. Wade, R. M. Wagner, and R. E. Williams. S. Starrfield is grateful to Drs. S. Colgate, A. N. Cox, C. F. Keller, M. Henderson, and K. Meyer for the hospitality of the Los Alamos National Laboratory and a generous allotment of computer time. This work was supported in part by NSF Grants AST85-16173 and AST88-18215 to Arizona State University and AST 86-11500 to the University of Illinois, by the Institute of Geophysics and Planetary Physics at Los Alamos, by NASA grants to Arizona State University and to the University of Colorado, and by the DOE.

\section{REFERENCES}

Gallagher, J. S., and Code, A. D., Astrophys. J., 189, p. 303, (1974).

Krautter, J., and Williams, R. E., Astrophys. J., 341, p. 968 (1989).

Robinson, E. L., Astron. J., 80, p. 515 (1975).

Shara, M. M., . Astron. Soc. Pac., 101, p. 5 (1989).

Starrfield, S., in Classical Novae, ed. M. Bode and A. Evans, New York, Wiley, p. 39, (1989).

Starrfield, S., Truran, J. W., Sparks, W. M., and Krautter, J., in The Future of EUV Astronomy, ed. R. Malina, in press (1989).

Starrfield, S., Truran, J. W., Sparks, W. M., and Kutter, G. S., Astrophys. J., 176, p. 169, (1972).

Truran, J. W., in Essays in Nuclear Astrophysics, ed. C. A. Barnes, D. D. Clayton, and D. Schramm, Cambridge, Cambridge University Press, p. 467, (1982). 